Poincare Journal of Analysis E Applications

Vol. 2014 (2), 63 - 70

CPoincare Publishers

\title{
SOME REMARKS ON ADOMIAN DECOMPOSITION METHOD
}

\author{
SILVIA SEMINARA ${ }^{\dagger}$ AND MARIA INÉS TROPAREVSKY
}

$\begin{array}{lll}\text { Date of Receiving } & : & 26.08 .2014 \\ \text { Date of Revision } & : & 09.12 .2014 \\ \text { Date of Acceptance } & : & 15.12 .2014\end{array}$

\begin{abstract}
Adomian Decomposition Method is a powerful method for solving general functional equations in Banach spaces. It provides a sequence of analytic approximate solutions to a wide range of nonlinear equations.

In this work we state the conditions under which, in the autonomous case, the approximate solutions to ordinary differential equations provided by Adomian Decomposition Method are the Taylor polynomials of the solution. We also offer an example where the approximations are not polynomials and highlight some limitations of the method.
\end{abstract}

\section{Introduction}

Adomian Decomposition Method (ADM), introduced by George Adomian in the '80s ([2], [3], [4]), is a powerful tool to solve a wide range of nonlinear equations. It produces analytical approximate solutions for very general nonlinear problems without linearization or simplification. The description of the method is simple, but it is difficult to give a general proof of convergence because of the wide range of problems it covers. The author gave no formal proof of the convergence but gave a justification based on the composition of Taylor series and offered lots of examples of application to problems with well known solutions, to show the goodness of the method. Since Adomian's first publications, a great number of works have appeared where ADM is proposed to solve functional, differential, integro-differential and algebraic equations, in a wide range of practical problems (see, for example, [7], [13], [16] and [20] as a small sample of them); also, many articles have been published comparing this method with other ones (we quote [12], [13] or [17], to give some few examples). Although it is theoretically possible to find approximate solutions to different type of equations by ADM, there exist some limitations and disadvantages that are not always mentioned when the method is applied. The limitations are mainly related to the strong hypothesis needed to assure the existence and convergence of the approximate

Key words and phrases. Nonlinear equations, approximate solutions, Adomian decomposition method, ODEs.

Communicated by. S.K. Kaushik

${ }^{\dagger}$ Corresponding author. 